\title{
Effect of yttrium content on the ultra-high cycle fatigue behavior of Mg-Zn-Y-Zr alloys
}

\author{
D.K. Xu ${ }^{1}$, E.H. Han ${ }^{1}$ \\ ${ }^{1}$ State Key Laboratory for Corrosion and Protection, Institute of Metal Research, Chinese Academy of Sciences, 62 \\ Wencui Road, Shenyang 110016, China \\ * Corresponding author: $\underline{\mathrm{dkxu} @ i m r . a c . c n}$
}

\begin{abstract}
In the super-long life regime, the fatigue behavior of as-extruded Mg-6wt\%Zn-xY-0.8wt\%Zr Mg alloys with Y content of 0,1, 2 and $3 \mathrm{wt} \%$ have been investigated, respectively. The result indicates that for all measured S-N curves, a plateau exists in the regime of $5 \times 10^{6}-10^{8}$ cyc, and then the fatigue strength gradually decreases between $10^{8}$ and $10^{9}$ cyc. Therefore, only fatigue strength corresponding to $10^{9}$ cyc can be determined. Compared with other alloys, the alloy with Y content of $2 \mathrm{wt} \%$ has the highest fatigue strength and its value is $105 \mathrm{MPa}$. SEM observations to fracture surfaces reveal that for all alloys, the fatigue crack mostly initiates at the surface or subsurface of samples failed within $10^{6}-10^{9}$ cyc. Further observation indicates that the crack initiation is related with activated slip bands instead of phase particles and activated twins. Based on the measured results and Murakami equation, it demonstrates that the fatigue strength of alloys is more dependent on the hardness values.
\end{abstract}

Keywords Mg alloy, super-long fatigue, slip band, fatigue properties

\section{Introduction}

Magnesium alloys are currently used in cars for low stress applications such as covers and less frequently for the mechanically loaded structural components such as wheels, transmission housings and pedals [1]. Generally, car wheels need to be stressed at different amplitudes for several $10^{8}$ cycles in service. For casting alloys, defects such as casting porosity and cavities are usually present and the fatigue properties are affected significantly by their shape and dimension [1-2]. Several material defects such as casting porosity, oxidation films and intermetallic inclusions, can act as crack initiation sites and reduce material's fatigue strength in the super-long fatigue life regime [1-2]. Therefore, the fatigue strength of as-cast Mg alloys corresponding to $10^{9}$ cycles is generally about 40-50 MPa [1]. In contrast, wrought alloys are basically defect-free and have superior mechanical properties, thus the evaluation of their fatigue properties is of great interest for understanding the intrinsic fatigue mechanism of Mg alloys [3]. In early reported work, researchers mainly focused on the fatigue behavior of wrought Mg alloys with the fatigue lifetime less than $10^{7}$ сус $[2,4,5]$. However, as for the ultra-high cycle $\left(10^{7}-10^{9}\right.$ cуc) fatigue behavior of wrought $\mathrm{Mg}$ alloys, only a few research papers can be referred [6-8]. Additionally, when compared with other system Mg alloys, Mg-Zn-Y-Zr alloys are much stronger [9-10], and their tensile strength can reach up to $380 \mathrm{MPa}$. Previous work demonstrated that $\mathrm{Y}$ content can remarkably influence the microstructure and tensile properties of Mg-Zn-Y-Zr system alloys [9-12]. Therefore, it can be predicted that the change of $Y$ content should have some effect on the fatigue behaviour of Mg-Zn-Y-Zr system alloys. However, so far, no related literature can be referred. The aim of this work is to disclose the crack initiation mechanism and to establish the relationship between $\mathrm{Y}$ content and the fatigue strength in the gigacycle regime by investigating the fatigue behavior of Mg-5.65\%Zn-xY-0.8\%Zr alloys with Y content of 0, 1, 2 and 3 wt\%, respectively.

\section{Experimental procedure}

The materials used in this study were the as-extruded Mg-Zn-Y-Zr alloys with different $\mathrm{Y}$ contents, which were prepared by special technology in magnesium alloy research department of 
IMR, China. Through inductively coupled plasma atomic emission spectrum (ICP-AES) apparatus, the chemical compositions of alloys I-IV were determined, as listed in Table 1. The extrusion ratio was 10:1. Vickers hardness testing was performed with a load of 250g (HV).

Table 1 Chemical composition of the as-extruded Mg-Zn-Y-Zr alloys

\begin{tabular}{ccccccc}
\hline Nominal & \multicolumn{5}{c}{ Composition (wt \%) } & HV \\
\cline { 2 - 5 } alloy & Mg & Zn & Y & Zr & Zn/Y & \\
Alloy I & Bulk & 5.68 & 0 & 0.78 & ----- & $62 \pm 5$ \\
Alloy II & Bulk & 5.53 & 1.08 & 0.83 & 5.12 & $78 \pm 4$ \\
Alloy III & Bulk & 5.64 & 1.97 & 0.73 & 2.86 & $74 \pm 4$ \\
Alloy IV & Bulk & 5.49 & 3.08 & 0.82 & 1.78 & $68 \pm 4$ \\
\hline
\end{tabular}

The specimens used for the super-long fatigue study were taken along extrusion direction (ED) of the plates. The dimensions of the specimens for fatigue test are shown in Fig. 1. Fatigue test was conducted on a Shimadazu USF-2000 ultrasonic fatigue testing machine at a resonance frequency of $20,000 \mathrm{~Hz}$, with a resonance interval of $150 \mathrm{~ms}$ and a stress ratio of $\mathrm{R}=-1$ in ambient air (temperature of $25-35{ }^{\circ} \mathrm{C}$, relative humidity of $40-60 \%$ ). Specimens were cycled at constant amplitude until failure or until at least $10^{9}$ cycles were reached. After test, fracture surfaces of failed samples were examined using Environmental Scanning Electron Microscope (XL30-FEG-ESEM).

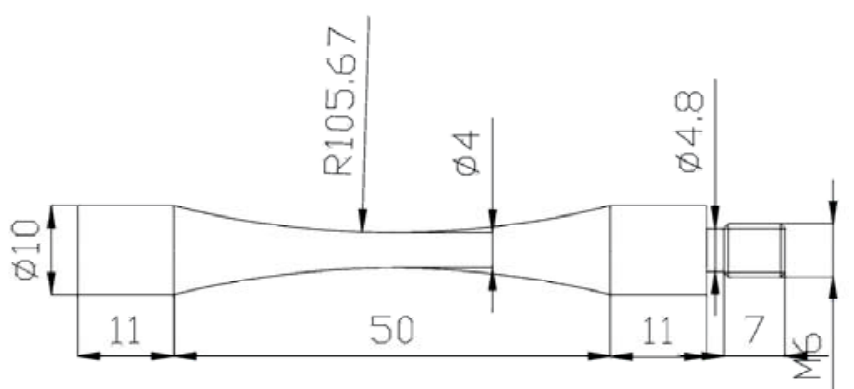

Fig. 1. Dimensions of the ultrasonic fatigue specimen.

\section{Results and discussion}

Fig. 2 shows the fatigue strength (S) versus lifetime (N) curves of four Mg-Zn-Y-Zr alloys. It can be seen that for all measured S-N curves, a plateau exists in the regime of $5 \times 10^{6}-10^{8}$ cyc, and then the fatigue strength gradually decreases between $10^{8}$ and $10^{9}$ cyc. Therefore, only fatigue strength corresponding to $10^{9}$ cyc can be determined. Compared with other alloys, the alloy with Y content of $1 \mathrm{wt} \%$ has the highest fatigue strength and its value is $105 \mathrm{MPa}$.

To understand and compare the crack initiation mechanism of different alloys that failed in the super-long fatigue regime, four representative samples designated as S1, S2, S3 and S4 are chosen for analysis, as shown in Fig. 2. Fig. 3 shows the overall fracture surfaces of samples. It can be seen that for all samples, the fatigue crack preferentially initiates at the surface or subsurface.

Based on the backscattered electron image, the overall fracture surface can be divided into three regions, i.e. crack initiation region (Region 1), steady crack propagation region (Region 2) and tearing region (Region 3), as shown in Fig. 4. It is very interesting to find that basically no phase 
particles exist in Region 1, whereas phase particles can be easily observed in Regions 2 and 3. Moreover, the number density of existing phase particles in Region 3 is obviously higher than that in Region 2. Therefore, it suggests that the existence of phase particles can not cause the fatigue crack initiation, but can act as the preferential route for crack propagation. Fig. 5 shows the optical microstructure of the areas just underneath the fracture surface of the sample S3. It reveals that only a few twins can be activated in the Region 1, whereas the activated twins can be easily observed in Regions 2 and 3. Additionally, density of the activated twins in Region 3 is remarkably higher than that in Region 2. In the same way, it indirectly demonstrates that the crack initiation is can not be caused by the activated twins under a stress amplitude far less than the yield stress. After crack initiation, the propagated crack will gradually increase the stress intensity factor near the crack tip, leading to the local plastic deformation and the activation of twins.

Previous work indicated that the micro cracks mainly initiate along the intense slip bands in the grain interior [12], as shown in Fig. 6. Therefore, it firmly demonstrates that the localized slip bands activated at elastic stress amplitude is the main reason for the crack initiation.
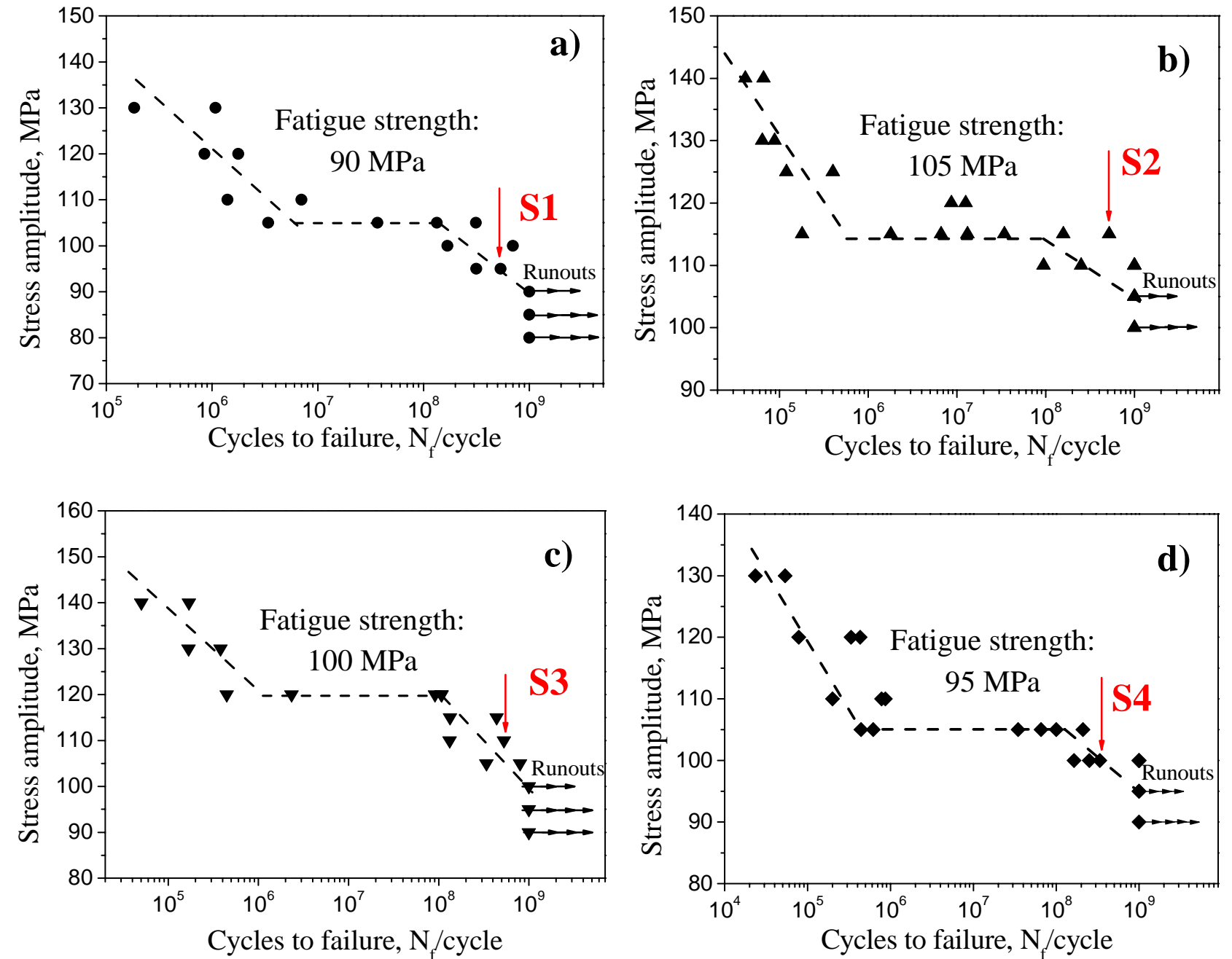

Fig. 2. S-N curves of the as-extruded Mg-Zn-Y-Zr alloys with Y content of: a) 0wt\%, b) 1wt\%, c) $2 w t \%$ and d) $3 w t \%$. 
Certainly, if the sample surface contains some scratches, it will further increase the occurrence possibility of crack initiation. Generally, the relationship between the "crack initiation area" size and fatigue strength or fatigue limit $\left(\sigma_{-1}\right)$ proposed by Murakami can also be used for Mg alloys, which can be expressed as [13]:

$$
\sigma_{-1}=\beta \frac{(\mathrm{HV}+120)}{\left(\sqrt{\mathrm{A}_{\mathrm{in}}}\right)^{1 / 6}}
$$

Where $\mathrm{HV}$ is the Vicks Hardness of Mg matrix, $\beta$ is a coefficient and $\mathrm{A}_{\text {in }}$ is the crack initiation area.
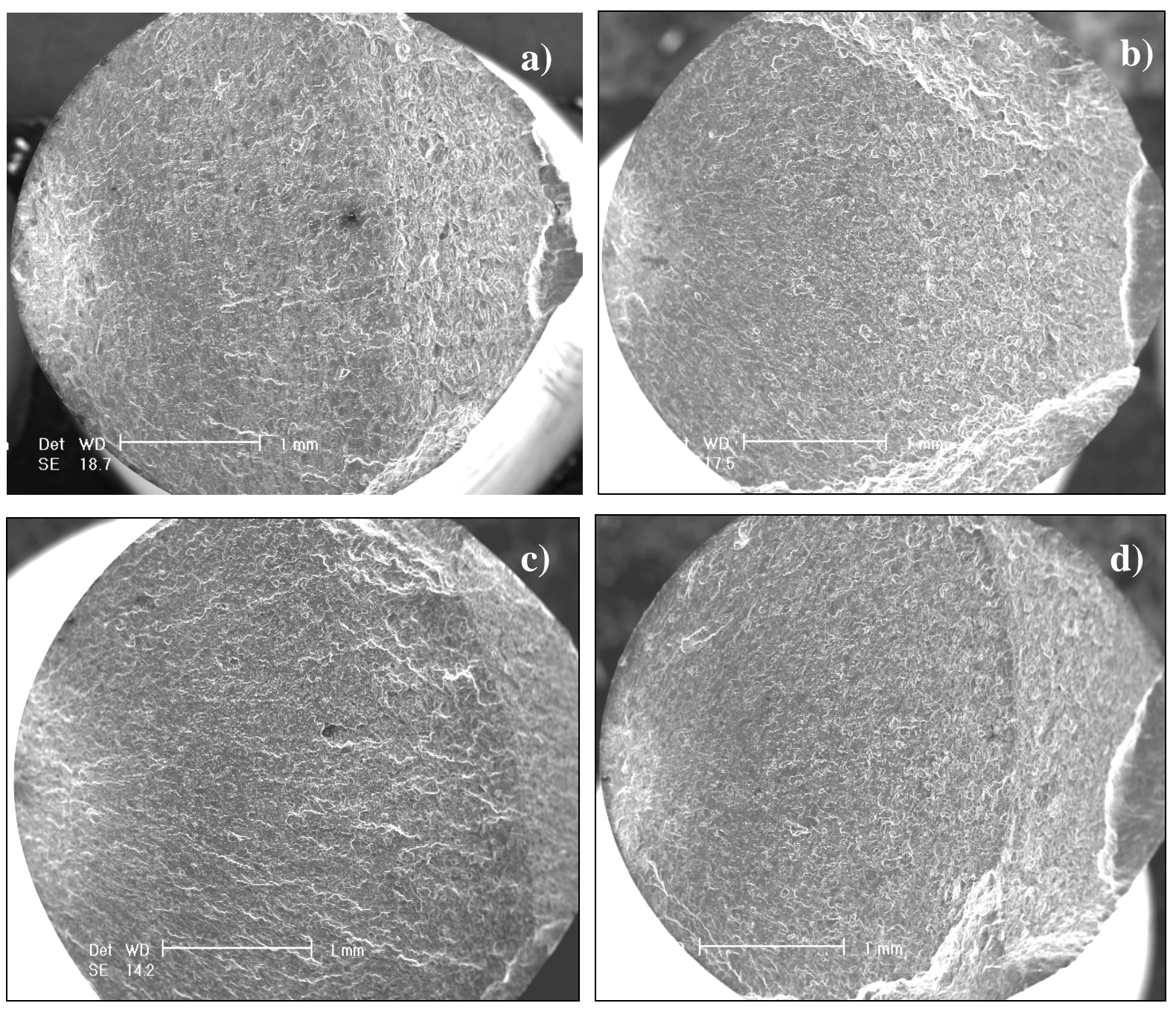

Fig. 3. Secondary electron images showing the overall fracture surfaces of samples: a) S1 (95MPa for 5E8 сус), b) S2 (110MPa for 5.2E8 сус), c) S3 (105MPa for 5.1E8 сус) and d) S4 (100MPa for $5.3 \mathrm{E} 8$ сус)

From Fig. 3, it can be seen that the crack initiation area for all failed samples is very similar. Then, the fatigue strength of alloys should be more dependent on the hardness values. Based on the results in Table 1, the hardness of the alloy with $\mathrm{Y}$ content of $1 \mathrm{wt} \%$ is relatively higher. According to Equation (1), the calculated fatigue strength corresponding to $10^{9}$ cycles should be higher than that of others, which is consistent with the measured results (Fig. 2). 


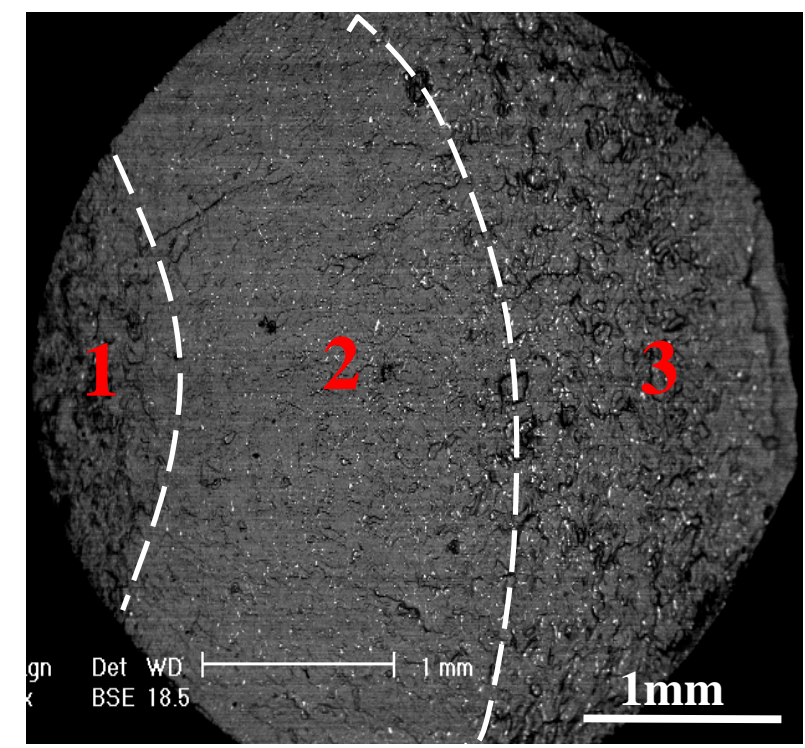

Fig. 4. Backscattered electron image showing the phase particle distribution on the fracture surface (S3).
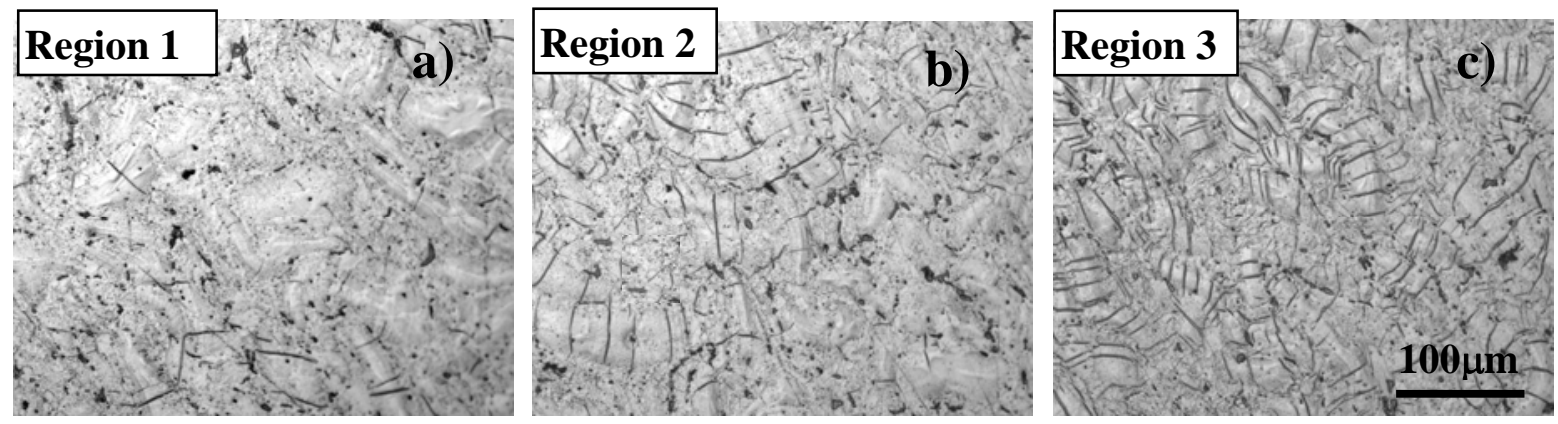

Fig. 5. Optical images of the as-polished fracture surface of sample S3 taken from regions 1-3 in Fig. 4.

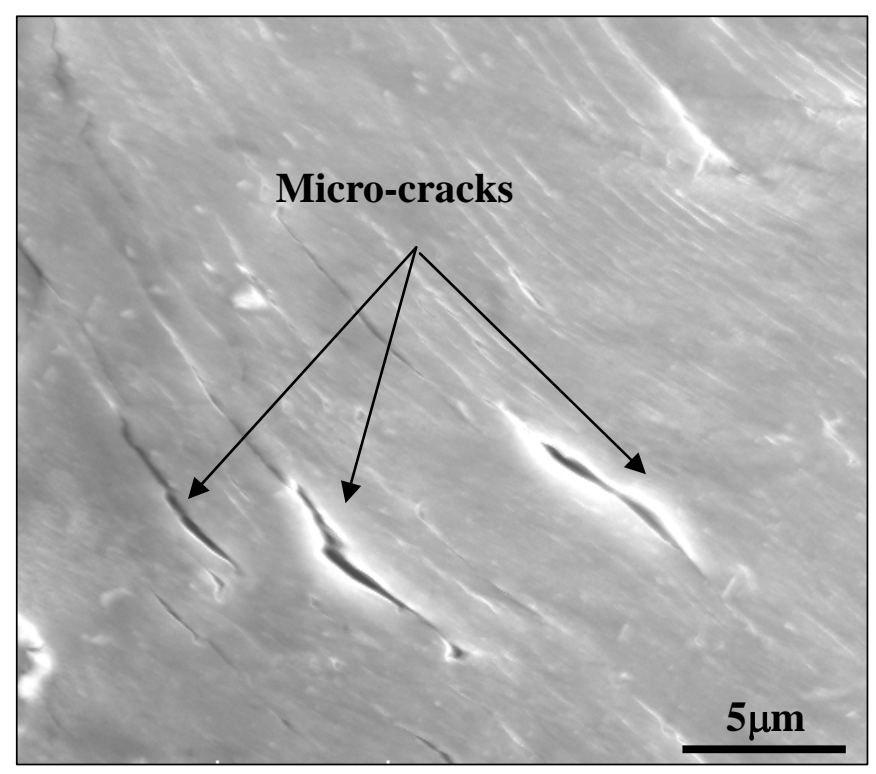

Fig. 6. Micro crack initiation at slip bands in the grain interior. [12] 


\section{Conclusions}

For all measured S-N curves, a plateau exists in the regime of $5 \times 10^{6}-10^{8}$ cyc, and then the fatigue strength gradually decreases between $10^{8}$ and $10^{9}$ cyc. Therefore, only fatigue strength corresponding to $10^{9}$ cyc can be determined. Compared with other alloys, the alloy with Y content of $2 \mathrm{wt} \%$ has the highest fatigue strength and its value is $105 \mathrm{MPa}$. Additionally, for all alloys, the fatigue crack mostly initiates at the surface or subsurface in the super-long fatigue lifetime regime.

\section{Acknowledgements}

This work was supported by National Natural Science Foundation of China projects under Grant No. 51171192 and No. 51271183, a National Basic Research Program of China (973 Program) project under Grant No. 2012CB067425 and an Innovation Fund of Institute of Metal Research (IMR), Chinese Academy of Sciences (CAS).

\section{References:}

[1] H. Mayer, M. Papakyriacou, Influence of porosity on the fatigue limit of die cast magnesium and aluminium alloys. Int. J. Fatigue, 25 (2003) 245-256.

[2] G. Eisenmeier, B. Holzwarth, H. Mughrabi, Cyclic deformation and fatigue behaviour of the magnesium alloy AZ91. Mater. Sci. Eng. A, 321 (2001) 578-582.

[3] K. Tokaji, M. Kamakura, Fatigue behaviour and fracture mechanism of a rolled AZ31 magnesium alloy Int. J. Fatigue, 26 (2004) 1217-1224.

[4] Z.B. Sajuri, Y. Miyashita, Y. Hosokai, Y.J. Mutoh, Effects of Mn content and texture on fatigue properties of as-cast and extruded AZ61 magnesium alloys. Int. J. Mech. Sci, 48 (2006) 198-209.

[5] T.S. Shih, W.S. Liu, Y.J. Chen, Fatigue of as-extruded AZ61A magnesium alloy. Mater. Sci. Eng. A, 325 (2002) 152-162.

[6] D.K. Xu, L. Liu, Y.B. Xu, E.H. Han, The crack initiation mechanism of the forged Mg-Zn-Y-Zr alloy in the super-long fatigue life regime. Scripta. Mater, 56 (2007) 1-4.

[7] D.K. Xu, L. Liu, Y.B. Xu, E.H. Han, The micro-mechanism of fatigue crack propagation for a forged Mg-Zn-Y-Zr alloy in the gigacycle fatigue regime. J. Alloys. Comps, 454 (2008) 123-128.

[8] F. Yang, S.M. Yin, S.X. Li, Z.F. Zhang, Crack initiation mechanism of extruded AZ31 magnesium alloy in the very high cycle fatigue regime. Mater. Sci. Eng. A, 491 (2008) 131-136.

[9] Z.P. Luo, D.Y. Song, S.Q. Zhang, Stengthening effects of rare-earths on wrought Mg-Zn-Zr-RE alloys. J. Alloy. Compd, 230 (1995) 109-114.

[10] Z.P. Luo, D.Y. Song, S.Q. Zhang, Effect of heat-treatment on the stability of the quasi-crystal in a Mg-Zn-Zr-Y alloy. Mater. Lett, 21 (1994) 85-88.

[11] D.K. Xu, L. Liu, Y.B. Xu, E.H. Han, Effect of microstructure and texture on the mechanical properties of the as-extruded Mg-Zn-Y-Zr alloys. Mater. Sci. Eng. A, 443 (2007) 248-256.

[12] D.K. Xu, L. Liu, Y.B. Xu, E.H. Han, The fatigue behavior of I-phase containing as-cast 
Mg-Zn-Y-Zr alloy. Acta. Mater, 56 (2008) 985-994

[13] Y. Murakami, M. Endo, Proc. The Behavior of Short Fatigue Cracks, EGF, Mech Engng Pub, London, 1986. 